\title{
Gamonwirkungen bei Chlamydomonas eugametos
}

\author{
Von Herbert Förster und Lutz Wiese \\ Aus dem Max-Planck-Institut für Biologie, Abt. H a r t m a n n, Tübingen \\ (Z. Naturforschg. 9 b, 548-550 [1954]; eingegangen am 26. Mai 1954)
}

\begin{abstract}
Die von Moewus 1933 beschriebene agglutinierende Wirkung geschlechts-spezifischer Gamone wird bestätigt. Die Lichtabhängigkeit der Abgabe und Wirkung dieser Gamone wird beschrieben. Vom Gesichtspunkt der neuen Befunde werden die später von M o e wu s beschriebenen lichtabhängigen Gamonwirkungen diskutiert.
\end{abstract}

\section{Die agglutinierend wirkenden G a mone}

A Agar kultivierte Zellen von Chlamydomonas eugametos werden kopulationsfähig, wenn man sie in ein flüssiges Medium überführt. Bringt man Zellen von beiden Geschlechtern zusammen, so bilden sich die für die Kopulation typischen Gruppen. Die Gruppen lösen sich nach einiger Zeit in isolierte Kopulationspärchen auf. Zentrifugiert oder filtriert man die Suspension kopulationsbereiter Zellen, so induziert das zellfreie Medium Gruppenbildung bei kopulationsbereiten Zellen des entgegengesetzten Geschlechts. Die Zellen geben also im kopulationsbereiten Zustand ein geschlechts-spezifisches Gamon an die Kulturflüssigkeit ab. Diese Filtratwirkung wurde von M o e w u s (1933) beschrieben ${ }^{1}$. Wir konnten sie ständig reproduzieren. In Einzelheiten unterscheiden sich unsere Ergebnisse von den Befunden, die Moewus beschreibt.

Nach M o e w u s sollen die Filtrate nur dann agglutinierend wirken, wenn Bakterien anwesend sind. Wir fanden dagegen auch die Filtrate bakterienfreier Kulturen agglutinierend wirksam. Auch beim Sterilfiltrieren durch Jenaer Glasfilter bleibt die Wirksamkeit erhalten. Das Filtrieren durch Glasfilter vermindert zunächst die Wirkung. Anscheinend wird die wirksame Komponente am Filter adsorbiert. Während die ersten $\mathrm{ccm}$, die das Filter passieren, oft völlig unwirksam sind, tritt beim Filtrieren einer größeren Menge nach einiger Zeit wieder optimale Wirksamkeit ein.

Die agglutinierende Gamonwirkung ist temperaturabhängig. Bei Zimmertemperatur $\left(19^{\circ} \pm 1^{\circ}\right)$ klingt die Wirksamkeit der Filtrate in 2-4 Tagen völlig ab. Ob das Filtrat belichtet oder unbelichtet auf-

1 F. M o ewus, Arch. Protistenkunde 80, 469-526 [1933]. bewahrt wird, ist gleichgültig. Im Eisschrank $\left(4^{\circ} \pm 1^{\circ}\right)$ bleibt die Wirksamkeit einige Wochen erhalten. Höhere Temperaturen zerstören die Wirkung schon nach kurzer Zeit. Die Tab. 1 zeigt die Größenordnung der Temperaturabhängigkeit. Das agglutinierende Gamon der $\hat{\jmath} \hat{\jmath}$ ist temperaturempfindlicher als das der 우.

Die agglutinierende Gamonwirkung geht im alkalischen Bereich $\left(p_{\mathrm{H}}>10\right)$ irreversibel verloren. Im sauren Bereich $\left(p_{\mathrm{H}} \cong 1\right)$ zeigt sich ein geschlechts-

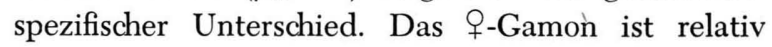
resistent, das $\hat{\delta}$-Gamon wird unwirksam. Der $p_{\mathrm{H}^{-}}$ Wert der Filtrate liegt normalerweise zwischen 6-7. Die Träger der agglutinierenden Gamonwirkung dialysieren in der Form, wie sie in den Filtraten vorliegen, nicht.

\section{Die Lichtabhängigkeit der Gamonwirkung}

Übergießt man $\hat{\delta}$-Kulturen und \&-Kulturen getrennt und belichtet sie, so werden die Zellen kopulationsfähig. Filtrate der belichteten Kulturen verursachen beim entgegengesetzten Geschlecht die Gruppenbildung. Jedes der Geschlechter gibt also im Licht sein Gamon ab. Außerdem wird jedes der Geschlechter im Licht reaktionsfähig auf das Gamon des anderen Geschlechts.

Übergießt man abends $\hat{\sigma}$-Kulturen und Q-Kulturen, stellt sie dunkel und zentrifugiert am nächsten Morgen im Dunkeln zellfrei, so ist das Zentrifugat der 우우 wirksam, das der $\hat{f} \hat{o}$ dagegen nicht. Erst nach Belichtung der $\hat{\delta}$-Kulturen wird ihr Zentrifugat wirksam.

Parallel dazu läuft die Reaktionsfähigkeit der Zellen auf die agglutinierenden Gamone. Die 우우 werden im Dunkeln voll reaktionsfähig: bei Zugabe von $\widehat{\delta}$-Filtrat bilden sich sofort Gruppen. Die $\hat{\delta}$-Dunkelzellen reagieren zunächst nicht auf das \&-Gamon; erst nach Belichtung werden sie reaktionsfähig.

Gibt man Q -Filtrat auf reaktionsbereite $\hat{\delta} \hat{\delta}$, so tritt sofort Gruppenbildung ein. Wird der Versuch im Licht durchgeführt, so beginnt die Agglutinations-Reaktion nach etwa 1 Stde. abzuklingen. Nach $4-5$ Stdn. ist sie ganz 


\begin{tabular}{|c|c|c|}
\hline \multirow{3}{*}{ Temperatur } & \multicolumn{2}{|c|}{$\begin{array}{c}\text { Zeit in Min. bis die Wirsamkeit } \\
\text { erloschen ist }\end{array}$} \\
& $0^{\top}$-Filtrat & ○-Filtrat \\
\hline & 300 & \\
$30^{\circ}$ & 180 & \\
$35^{\circ}$ & 60 & \\
$40^{\circ}$ & $<10$ & 90 \\
$50^{\circ}$ & & 20 \\
$60^{\circ}$ & & 8 \\
$80^{\circ}$ & & $<3$ \\
$90^{\circ}$ & & \\
$100^{\circ}$ & & \\
& & \\
\hline
\end{tabular}

Tab. 1. Beständigkeit der Filtrate bei höheren Temperaturen. (Die angegebenen Zeiten wurden nicht statistisch gesichert.)

erloschen. Die Reaktionsdauer ist von der zugegebenen Filtratmenge abhängig. Gibt man viel Filtrat auf wenig Zellen, so hält die Reaktion länger an. Gibt man wenig Filtrat auf viel Zellen, so klingt die Reaktion schneller ab. Die angegebenen Zeiten beziehen sich auf $1 \mathrm{ccm}$ Filtrat, das auf $1 \mathrm{ccm}$ Zellen von einer Dichte von $10^{6}$ Zellen/ccm gegeben wird. Die Reaktionsdauer der 우우 auf das $\widehat{\sigma}$-Filtrat ist kürzer. Sie beträgt unter den gleichen Bedingungen nur etwa 2-3 Stunden. Stellt man nach Abklingen der Reaktion wieder Zentrifugate her, so wirken diese nur noch zellspezifisch: das Zentrifugat der $ㅇ$ wirkt auf $\hat{\sigma} \hat{\delta}$, das Zentrifugat der $\hat{\delta} \hat{\delta}$ wirkt auf $\phi$ ㅇ․ Dagegen sind die Filtrate, die zum Induzieren der Agglutinations-Reaktion zugegeben wurden, nicht mehr nachweisbar. Die zugegebenen Gamone sind während der Reaktion aufgebraucht.

Werden belichtete, voll reaktionsfähige Zellen nach Filtratzugabe dunkel gestellt, so zeigen sich charakteristische Unterschiede sowohl zu den Verhältnissen im Licht wie zwischen den beiden Geschlechtern.

Gibt man O-Filtrat auf $\hat{\delta} \hat{\sigma}$, so geht die Gruppenbildungsreaktion im Dunkeln sehr bald zurück, d. h. die $\hat{\delta}-$ Zellen verlieren im Dunkeln sehr schnell ihre Reaktionsfähigkeit auf das $ᄋ$-Gamon, und das -G-Gamon wird nicht aufgebraucht. Stellt man nach einigen Stdn. Zentrifugate her, so ist darin das $Q$-Gamon noch nachweisbar: Das Zentrifugat wirkt wieder auf belichtete $\hat{\sigma}$-Zellen. Nach der gleichen Zeit ist im Hellversuch das O-Gamon nicht mehr nachweisbar.

Gibt man dagegen $\widehat{\delta}-F i l t r a t$ auf $q Q q$, so läuft die Agglutinations-Reaktion im Dunkeln weiter. Gegenüber der Reaktion im Licht ist sie nur geringfügig schwächer. Das $\hat{\sigma}$-Gamon wird auch im Dunkeln aufgebraucht: stellt man von den behandelten $\bigcirc \bigcirc$ nach einigen Stunden Zentrifugate her, so wirken sie zellspezifisch nur auf $\hat{\sigma} \hat{\delta}$, nicht auf $q 0$.

\section{Diskussion}

In der Arbeit von 1933 hat M o ew us die agglutinierende Gamonwirkung und den Einfluß des Lichts auf die Kopulationsfähigkeit beschrieben. Übereinstimmend mit unseren Versuchen stellte er fest, daß die Träger der agglutinierenden Gamonwirkung thermolabil und photostabil sind. Außerdem beschreibt er - nicht klar abgegrenzt von der agglutinierenden Gamonwirkung - eine weitere Gamonwirkung, die im Mittelpunkt seiner späteren Arbeiten steht. Zur Deutlichkeit bezeichnen wir die agglutinierenden Gamone mit „A“, die weiterhin von Moewus beschriebene 2. Gamonwirkung mit „B“. Moewus hat die Gamonwirkung B erfaßt, indem er auf kopulationsunfähige Dunkelzellen Filtrate von kopulationsfähigen Zellen des gleichen Geschlechts gab. Unter dem Einfluß des Filtrats werden nach Moewus die Zellen ohne Belichtung kopulationsfähig. Das Filtrat sollte also Stoffe enthalten, die von den Zellen normalerweise nur im Licht gebildet werden und die für die Kopulationsfähigkeit entscheidend sind. Diese Gamone sollen im Gegensatz zum Träger der agglutinierenden Gamonwirkung photolabil sein. Sie wurden als geschlechts-spezifische $\mathrm{Ge}-$ mische von cis- und trans-Crocetindimethylester angesehen.

Die durch das Filtrat bedingte Kopulationsfähigkeit der Dunkelzellen wurde von Moewus durch Zugeben von kopulationsfähigen Zellen des anderen Geschlechts getestet. In der Annahme, daß die Gamonwirkung A an die Anwesenheit von Bakterien gebunden sei, konnte Moewus die bei diesen Testen auftretende Gamonwirkung A unberücksichtigt lassen, wenn er mit bakterienfreien Kulturen arbeitete. Auf Grund unseres Befundes, daß auch bakterienfreie Filtrate wirken, ist ein Test auf die Gamonwirkung B zumindest erschwert, da sich immer die Gamonwirkung A bemerkbar macht. Gibt man z. B. auf kopulationsunfähige \$-Dunkelzellen wirksames $\hat{\delta}$-Filtrat und testet dann diese Dunkel$\hat{\jmath} \widehat{\jmath}$ gegen reaktionsfähige $ㅇ ㅜ$, so tritt zwangsläufig allein auf Grund der Gamonwirkung A Gruppenbildung ein, denn bei den 우우 wird durch das $\hat{\delta}$-Filtrat eine Isoagglutination hervorgerufen. Es ist also auf diese Weise im Test auf Gruppenbildung schwierig, eine Gamonwirkung B nachzuweisen, da immer die Gamonwirkung A auftritt und eine Gamonwirkung B gar nicht isoliert in Erscheinung treten kann.

Es scheint auch nicht möglich zu sein, die Gamonwirkung B am nächsten Kopulationsstadium, der Pärchenbildung, zu testen. Die Pärchenbildung tritt nur bei dem Zusammengeben von Zellen beider Geschlechter auf. Damit sind allein für die Agglutinations-Reaktion vier Faktoren im Spiel, die alle für den Gesamtverlauf der Kopulation wesentlich sind 
(Bildung von $q$-Gamon $\mathrm{A}=\mathrm{A}+$, Bildung von $\hat{\jmath}-\mathrm{Ga}-$ mon $\mathbf{A}=\mathbf{A} \hat{\delta}$, Reaktionsfähigkeit der $+q$ auf $\mathbf{A} \hat{\delta}$, Reaktionsfähigkeit der $\hat{\delta} \hat{o}$ auf Aㅇ). Zwei dieser Faktoren, nämlich die Abgabe von A $\hat{\delta}$ und die Reaktionsfähigkeit der $\hat{\delta} \hat{\delta}$ auf $\mathrm{A}+\mathrm{q}$ sind lichtabhängig und verdecken daher eine etwa vorhandene lichtabhängige Gamonwirkung B. Unsere Versuche über den Einfluß des Lichtes auf die Kopulation ${ }^{2}$ machen die Annahme eines weiteren lichtabhängigen Kopulationsfaktors (Gamon B) überflüssig. Sie sind durch die Lichtabhängigkeit der Gamonwirkung A vollständig zu erklären. Die $q$ $\uparrow$ brauchen kein Licht, um kopulationsfähig $\mathrm{zu}$ werden, für die Kopulationsfähigkeit der $\widehat{\jmath} \hat{o}$ ist dagegen das Licht ein wichtiger Faktor. Diese Lichtabhängigkeit ist zwar nicht obligatorisch, da Kopulationen prinzipiell auch im Dunkeln möglich sind. Wenn man die zusammengegebenen Zellen von beiden Geschlechtern längere Zeit stehen läßt, treten meist auch im Dunkeln Gruppen und Pärchen auf. Welche Faktoren hierbei an Stelle der Lichtwirkung treten, ist noch ungeklärt. Der Kopulationsverlauf ist aber im Dunkeln mindestens um mehrere Stdn. verzögert.

Besonders aufschlußreich sind die Licht-Dunkel-

2 H. F örster u. L. Wi es e, Z. Naturforschg. 9 b, 470 [1954].
Versuche an $\hat{\jmath} \hat{\jmath}$. Im Licht voll reaktionsfähig gewordene $\hat{\jmath} \hat{\jmath}$ verlieren beim Übertragen ins Dunkle ihre Reaktionsfähigkeit auf das A -Gamon. Übereinstimmend damit ist auch die Pärchenbildung von Kulturen, die im Licht voll kopulationsfähig geworden sind und dann im Dunkeln zusammengegeben werden, stark verzögert gegenüber den Kontrollen. Die Gamone Aô und $A \hat{q}$ sind bereits im Licht abgegeben, und sie bleiben nach Dunkelstellen wirksam vorhanden. Diese Versuche stehen im Widerspruch zu den Filtratversuchen von Moewus. Nach seinen Versuchen sollen Filtrate kopulationsfähiger Zellen das Licht ersetzen, indem sie die Zellen im Dunkeln kopulationsfähig machen. Das Gamon B müßte danach im Dunkeln wirksam bleiben. In unseren Versuchen nimmt aber die Kopulationsfähigkeit nach Dunkelstellen sehr rasch ab. Dieser Effekt läßt sich ohne weiteres auf das Verhalten der $\hat{\jmath} \hat{\jmath}$ gegenüber Gamon A우 zurückführen. Testet man allerdings solche $\hat{\jmath} \hat{b}$, die im Licht reaktionsfähig geworden sind und dann dunkel gestellt wurden, gegen reaktionsfähige Hell- +9 , so tritt sofort Gruppenbildung ein.

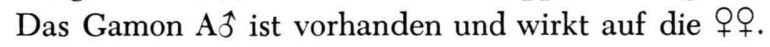
Bei der Beobachtung unter dem Binokular werden die $\widehat{\delta}$-Zellen belichtet und infolgedessen nach kurzer Zeit wieder voll kopulationsfähig.

\title{
Der kontraktile Myosinfaden aus glatter Muskulatur
}

\author{
Von Dieter Dörr und Hildegard Portzehl \\ Aus dem Physiologischen Institut der Universität Tübingen \\ (Z. Naturforschg. 9 b, 550-555 [1954]; eingegangen am 15. Juli 1954)
}

1. Aus dem glatten Fußmuskel von Anodonta lassen sich in ähnlicher Weise geordnete Fäden aus mehrfach gereinigtem Aktomyosin herstellen wie aus den quergestreiften Muskeln des Kaninchens.

2. Diese Fäden haben mit den Aktomyosinfäden aus Kaninchenmuskeln das niedrige Spannungsmaximum, die niedrige Zerreißfestigkeit und die sehr geringe Verkürzungsgeschwindigkeit gemeinsam. In diesen drei Eigenschaften unterscheiden sich beide Arten der Fadenmodelle von den Fasermodellen, die aus den gleichen Muskeln hergestellt sind.

3. Ferner zeigen die Fadenmodelle aus Anodonta das gleiche Verkürzungsmaximum, die gleiche Empfindlichkeit gegen Salyrgan und die gleiche Abhängigkeit der Kontraktion von der ATP-Spaltung und der Erschlaffung von der Hemmung der ATP-Spaltung wie alle Arten von Faser- und Fadenmodellen.

4. Die Fadenmodelle aus Anodonta-Aktomyosin unterscheiden sich von den Fadenmodellen aus Kaninchen-Aktomyosin in der Temperaturabhängigkeit der Spannung in der Unvollständigkeit der Einstellung des Spannungsgleichgewichts, wenn die gleiche Temperatur einmal von oben und einmal von unten erreicht wird, in der Lage des ATP-Optimums der Kontraktion und ihrer Beeinflußbarkeit durch Pyrophosphat.

5. Da beide Fadenmodelle sich in den letztgenannten Eigenschaften ebenso verhalten wie die Fasermodelle aus den gleichen Muskeln, müssen diese Eigenschaften auf Besonderheiten des jeweiligen Aktomyosins beruhen und nicht auf Besonderheiten der übergeordneten Struktur der Muskeln, die nur in den Fasermodellen noch erhalten ist.

* Mit Unterstützung des Unitarian Service Committee und des Oberlaendertrust, Philadelphia. 